\title{
FOCUS ON CELL BIOLOGY OF VIRUS-PLANT AND VIRUS-VECTOR INTERACTIONS
}

\section{MPMI Focus Issue Overview}

A successful viral infection requires complex, compatible molecular interactions between the invading virus and the host. A better understanding of such interactions may assist in the development of novel approaches to control viral diseases for sustainable crop production. In the past decade, the cell biology of virus-host and virus-vector interactions has been one of the most exciting areas of research in the molecular plant-microbe field. This is partially attributed to the availability of powerful cell biology techniques, including imaging tools like confocal microscopy and electron microscopy and tomography. As a result, there has been an unprecedented increase in knowledge in the areas of the bi- and tripartite interactions of virus, host, and vector. We now have a much clearer picture of viral virulence mechanisms, virus-induced host defenses, viral counteracting strategies, and viral circulations in the insect vectors. This Focus Issue highlights molecular virus-plant and virus-vector interactions in the areas of cell biology and closely related disciplines and explores biotechnology-based antiviral strategies using knowledge generated from these research areas.

Among major plant pathogens, viruses are obligate intracellular pathogens that can infect all field and greenhouse crops. Viral pathogens account for nearly $50 \%$ of newly emerging plant diseases and are considered a major constraint to agriculture, threatening global food security. Viruses have small genomes with very limited coding capacity and must therefore depend on host cellular machineries and various factors to complete their infection processes and life cycle. On one hand, viruses induce extensive reprograming of infected cells, including but not limited to proliferation of membranes and membranous vesicles and modification of the cellular organelles, leading to the formation of a favorable infection microenvironment. On the other hand, virus infection activates plant defense mechanisms to restrict and suppress virus replication and spread. In response, viruses have evolved corresponding counteracting strategies. Altogether, these molecular interplays have a profound impact on plant physiology and development, leading to transcriptomic, proteomic, metabolic, and morphological changes and the outcome of symptom development in infected plants.

In plant tissues, macromolecules or complexes cannot move freely between cells due to the cell-wall barrier. Plasmodesmata (PD), specialized intercellular organelles that cross the cell wall to establish cytoplasmic and endomembrane continuity between adjacent cells, allow small molecules to traffic freely between cells. To move from the primarily infected cell to neighboring cells, viruses must pass through PD. In this issue, Reagan and Burch-Smith review the way in which viruses manipulate PD and alter PD structure for intercellular movement and how this knowledge is used to interpret native PD function. They also address one important but less well studied area, i.e., how viruses move intracellularly to and through PD, by discussing the possible role of the cytoskeleton in these processes.

To establish systemic infection, viruses must have the ability to undergo long-distance transport to reach remote tissues. This long-distance spread occurs via the phloem. Previously, very little has been done to understand how phloem tissues respond to virus infection. Potyviruses represent the largest group of known plant viruses and include several agriculturally important members, such as plum pox virus (PPV). Collum et al. report their exciting findings from studies directed towards identifying phloem- and nonphloemspecific gene responses to PPV infection, using translating ribosome affinity purification followed by RNA sequencing. Their data provide new insights into temporal and spatial coordination of defense responses in phloem tissues to virus infection. It is worth mentioning that PPV, as one of the top 10 mostimportant plant viruses, is among the few best-studied plant viruses. Rodamilans et al. summarize current knowledge about PPV-host interactions and present a comprehensive overview of the relevance of these interactions to viral virulence and plant survival and resistance.

In view of the role of the phloem in virus infection, the phloem may be a potential target for the control of viral diseases. Indeed, in this issue Tzean et al. demonstrate that a phloem-specific promoter can direct the expression of hairpin RNA effectively against tomato yellow leaf curl Thailand virus
(TYLCTHV), a DNA geminivirus. This work adds another example of RNAi conferring strong resistance to viral pathogens. More interestingly, they present evidence that wild-type susceptible scions may obtain TYLCTV resistance by grafting to RNAi rootstocks with the phloemspecific promoter but not the $35 \mathrm{~S}$ promoter, raising the possibility for the application of this phloem-specific RNAi approach to generate resistant rootstocks for the control of viral diseases in the nontransgenic scions for the production of transgene-free consumables. Consistent with this work, in another original research article, Tatineni et al. show that RNAi can be designed to simultaneously target two economically impactful wheat viruses, wheat streak mosaic virus and triticum mosaic virus. This research is important for wheat growers, particularly in the Great Plains region, where mixed infections by these viruses are often seen and synergistic interactions exacerbate yield losses. It would be interesting to test if the two approaches developed by Tezean et al. and Tatineni et al. can be integrated to simultaneously target multiple viral pathogens in nontransgenic scions.

It is well-known that different viruses, even different strains and isolates of the same virus may induce different symptoms. This is the case for the two strains of Sri Lankan cassava mosaic virus (SLCMV), SLCMV-HN7 and the Chinese strain SLCMV-Col. The latter is much more virulent than the former. In this focus issue Wang et al. reveal that a 7-amino acid motif of the replication protein is essential for virulence and is also responsible for the activation of salicylic acid (SA)-mediated defense response. This work suggests that the balance between virulence and host SA defense responses may regulate the progression of SLCMV infection.

The majority of plant-infecting viruses are RNA viruses. RNA metabolism is expected to be involved in virus infection. RNA granules, present in the cytosol or nucleus, are dense aggregations composed of proteins and RNAs. In recent years, accumulating evidence suggests that RNA granules play roles in RNA transcription, modification, processing, decay, translation, and arrest in eukaryotic cells and are involved in diverse biological processes, such as the immune response. In this issue, Xu et al. summarize recent progress in the understanding of the interplay of RNA granules with virus infection.

Many plant viruses need to be transmitted by a vector, most often insects such as leafhoppers and aphids. The virus-insect interaction remains a hot research topic. In this issue, Chen and Wei discuss current advances in the cell biology of virus-insect vector interactions including virus-induced inclusion bodies, changes of insect cellular ultrastructure, and immune response of insects to the viruses, especially autophagy and apoptosis, and point out future prospects of this research area.

In comparison with the virus-plant and virus-vector interactions, which have been extensively studied, we have just begun to understand the plantvirus-vector tripartite interactions. Lu et al. demonstrate a novel case of symbiotic virus-mediated three-species interaction. Symbiotic viruses exist in many insects and can be horizontally transferred to host plants during insect feeding. Lu et al. show that a symbiont virus of pea aphid promotes aphid adaptation to host plants by inhibiting the level of jasmonic acid. In another original research article, Shah et al. provide evidence that infection of winter oilseed rape by a mycovirus causes hypervirulence and induces systemic resistance to its vector, a fungal pathogen responsible for the devastating blackleg disease in oilseed rape. This study opens a promising avenue for the application of vector-borne viruses to protect plants from pathogen vectors by activation of defense mechanisms.

We are very pleased to have assembled this focus issue that addresses important research on our Focus Issue topic. We would like to express our sincere gratitude to the authors for their valuable contributions and the MPMI production team for their excellent professionalism and hard work that makes publication of this issue at this time.

Guest Editors

Aiming Wang, Tessa M. Burch-Smith, and Yi Li 